\title{
Complications Associated with the Percutaneous Insertion of Fiducial Markers in the Thorax
}

\author{
Nikhil Bhagat $\cdot$ Nicholas Fidelman $\cdot$ Jeremy C. Durack • \\ Jeremy Collins • Roy L. Gordon · Jeanne M. LaBerge • \\ Robert K. Kerlan Jr.
}

Received: 14 September 2009/Accepted: 17 June 2010/Published online: 27 July 2010

(c) The Author(s) 2010. This article is published with open access at Springerlink.com

\begin{abstract}
Purpose Radiosurgery requires precise lesion localization. Fiducial markers enable lesion tracking, but complications from insertion may occur. The purpose of this study was to describe complications of fiducial marker insertion into pulmonary lesions.

Materials and Methods Clinical and imaging records of 28 consecutive patients with 32 lung nodules or masses who underwent insertion of a total of 59 fiducial markers before radiosurgery were retrospectively reviewed.

Results Eighteen patients (67\%) developed a pneumothorax, and six patients (22\%) required a chest tube. The rates of pneumothorax were $82 \%$ and $40 \%$, respectively, when 18 -gauge and 19-gauge needles were used for marker insertion $(P=0.01)$. Increased rate of pneumothorax was also associated with targeting smaller lesions $(P=0.03)$ and tumors not in contact with the pleural surface $(P=0.04)$. A total of 11 fiducials (19\%) migrated after insertion into the pleural space (10 markers) or into the airway (1 marker). Migration was associated with shorter distances from pleura to the marker deposition site $(P=0.04)$ and with fiducial placement outside of the target lesion $(P=0.03)$.

Conclusion Fiducial marker placement into lung lesions is associated with a high risk of pneumothorax and a risk of fiducial migration.
\end{abstract}

Keywords Fiducial marker - Complications .

Pneumothorax $\cdot$ Migration $\cdot$ Lung neoplasm

\footnotetext{
N. Bhagat · N. Fidelman $(\bowtie) \cdot$ J. C. Durack · J. Collins .

R. L. Gordon · J. M. LaBerge · R. K. Kerlan Jr.

Department of Radiology, University of California,

San Francisco, 505 Parnassus Avenue, Room M-361,

San Francisco, CA 94143, USA

e-mail: Nicholas.Fidelman@ radiology.ucsf.edu
}

\section{Introduction}

Radiosurgery was first developed for delivery of high radiation doses to well-circumscribed brain metastases [1-4]. Consistent doses could be delivered to these lesions because of their relatively stationary position. When this technique is modified to treat tumors located elsewhere in the body, accurate delivery of consistent radiation doses becomes difficult as a result of tumor motion during respiration. This problem is especially evident in the lung.

Commercially available radiosurgery systems rely on differences in tissue density for lesion tracking. The inherent difference in density between the tumor and the surrounding tissues may be sufficient for the system to track lesion movement during respiration. When tumor tissue density is not sufficiently differentiated from the density of surrounding normal parenchyma, a metallic fiducial marker placed within or adjacent to the lesion is required for accurate radiotherapy targeting.

Complications from fiducial marker insertion have been reported, including pneumothorax in 33-68\% of patients as well as bleeding [5-7]. Fiducial markers may also migrate into the pleural space, the airway, or into the vascular structures. The purpose of this study was to describe complications of fiducial marker placement into pulmonary lesions.

\section{Materials and Methods}

This study was approved by the Committee on Human Research at our institution. Informed consent requirement was waived. Records of 28 consecutive patients (15 men, 13 women; mean age 64 years) referred to our institution for percutaneous fiducial marker placement before 
initiation of cyber knife radiosurgery (Accuray, Sunnyvale, CA) between December 2004 and October 2009 were retrospectively reviewed. Indications for radiosurgery included unresectable non-small-cell lung carcinoma ( $n=14$ patients) and metastatic disease to the lungs from colorectal carcinoma $(n=5)$, soft tissue sarcoma $(n=5)$, melanoma $(n=2)$, renal-cell carcinoma $(n=1)$, and lymphoma $(n=1)$. One male patient with two nodules in the left lung underwent a computed tomographic (CT)guided biopsy of one of the nodules $24 \mathrm{~h}$ before fiducial placement. This patient developed a pneumothorax at the time of biopsy, which was treated by insertion of a chest tube. The chest tube remained in place at the time of the fiducial placement. Data from this patient were therefore excluded from the analysis of pneumothorax-related risk factors, but were included in the analysis of fiducial migration.

At the request of our institution's radiation oncologists, between one and three fiducials, each measuring $1.2 \mathrm{~mm}$ by $3 \mathrm{~mm}$ (Alpha-Omega Services, Bellflower, CA), were placed within or adjacent to each lesion. The number of fiducials used in each case was at the discretion of the interventional radiologist performing the procedure. In general, lesions greater than $3 \mathrm{~cm}$ in diameter were targeted with two or three markers, whereas one fiducial was used for nodules less than $3 \mathrm{~cm}$ in diameter. The fiducials did not have to be positioned within the lesion. Marker placement within $1 \mathrm{~cm}$ of the lesion was acceptable for targeting. If a fiducial migrated away from its intended location, additional markers were inserted until at least one marker remained within or adjacent to the target lesion.

A total of 59 fiducial markers were placed. In four patients, two lesions located in the same lung were targeted; one lesion was targeted in the remainder of the patients. Because marker migration occurred upon insertion in six patients, up to seven fiducials (range 1-7) were required to achieve durable lesion targeting with at least one marker. Between one and seven fiducials were used for each lesion (median 1), and between one and seven fiducials were used for each patient (median 2). Lung biopsies were not performed before fiducial placement.

In each insertion procedure, CT was used to guide a 15-cm, 18-gauge double-wall Chiba needle (18 patients; Cook, Bloomington, IL) or a 15-cm 19-gauge Temno guiding double-wall needle (10 patients; Cardinal Health, Dublin, $\mathrm{OH}$ ) into the lung lesion (Fig. 1). Choice of needle gauge was at the discretion of the physician operator. After the needle was placed in the lesion, the marker was delivered through the needle with a 0.035 -inch straight guide wire (Long-Tip; Cook) or the needle stylet. After the procedure, patients were followed with chest radiographs at hourly intervals for $2 \mathrm{~h}$. Chest tube insertion was performed in patients with a pneumothorax and one or more of

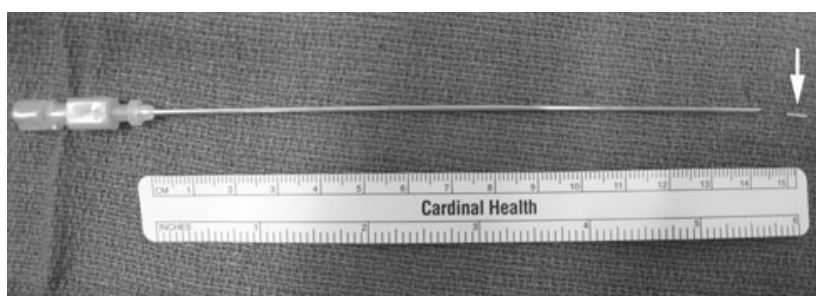

Fig. 1 Standard apparatus for fiducial insertion: 15-cm, 18-gauge double-wall needle and a gold seed fiducial marker (arrow)

the following clinical features: dyspnea, oxygen saturation of $<92 \%$ on room air, pneumothorax exceeding $25 \%$ of hemithorax volume, or increasing pneumothorax on serial radiographs.

Patient records including CT scans of the thorax, chest radiographs, and clinic notes were retrospectively reviewed. Follow-up chest CT scans and/or chest radiographs were available for all patients. Follow-up period ranged from 1 to 46 months (mean 11.9 months). Preprocedure CT images were used to determine lesion volume and to look for radiographic evidence of emphysema (presence of bullae or parenchymal lucencies located in the typical centrilobular, panlobular, or paraseptal distribution pattern). Presence or absence of emphysema was also determined from chart reviews, which were focused on clinic notes, hospital discharge summaries, and on the results of pulmonary function tests. Patients were categorized as having emphysema if they carried this diagnosis in the medical record or if there was evidence of emphysema on CT. Postprocedural CT scans were also evaluated for the length of needle trajectory through lung parenchyma, fiducial marker position in relation to the lesion, and development of a pneumothorax. The number of needle adjustments within the lung parenchyma (defined as any needle manipulation after crossing the pleura and documented by the procedural CT) before deposition of each fiducial was also determined. Needle adjustments before crossing the pleura were not counted. Targeting of each lesion required a median of one adjustment (range 1-8). The location of the fiducial markers on follow-up CT scans and chest radiographs was compared to their location on the initial scans in order to determine whether fiducial migration had occurred.

Our study focused on the rates of pneumothorax and fiducial migration as well as on elucidation of potential risk factors that may lead to these complications. Patients with and without these complications were compared with regard to several factors including lesion volume, length of needle trajectory through the lung parenchyma, number of fiducials inserted in a given lesion, number of needle adjustments within the lung parenchyma required for fiducial positioning, lesion location (upper or lower lung zone), lesion location in relation to the pleural surface, 
preinsertion needle tip location with respect to the target lesion (inside or outside the target lesion), presence or absence of pneumothorax before fiducial insertion, presence or absence of emphysema (on the basis of CT and clinical records), violation of a fissure, and needle gauge. The associations of the above factors with fiducial placement-related complications including pneumothorax, chest tube placement, and fiducial migration were analyzed by multivariate generalized estimating equation models. This modeling approach appropriately accounted for the statistical dependence among multiple fiducials and multiple lesions in the same individual. All analyses were conducted by SAS software, version 9.2 (SAS Institute, Cary, NC). All results were reported as $P$ values, with $P=0.05$ serving as a threshold for statistical significance. Lesion volumes were calculated by multiplying the cross-sectional area of each section by the section thickness and adding the individual section volumes. Length of needle trajectory through the lung parenchyma was measured at the point of fiducial deposition as the distance between needle tip and pleural surface. Because 10 fiducials migrated immediately or soon after their insertion, this measurement was used to estimate the distance from the fiducial insertion site to the pleural surface.

\section{Results}

Eighteen of 27 patients (67\%) developed a pneumothorax as a result of the fiducial marker insertion. Pneumothoraces were detected immediately after placement of the fiducials on the chest CT images (Fig. 2). Of the 18 patients with pneumothorax, six (33\% of patients with pneumothorax; $22 \%$ of all patients) required chest tube placement because of clinical symptoms or increasing size of the pneumothorax. Increased rates of pneumothorax and chest tube insertion were found when 18-gauge needles were used for fiducial marker placement (Table 1). Fourteen of 17 patients $(82 \%)$ developed pneumothoraces after fiducial insertion through an 18-gauge needle, compared to 4 of 10 patients $(40 \%)$ in whom 19-gauge needles were used $(P=0.01)$. In addition, the rate of postprocedure pneumothorax was $40 \%$ for lesions extending to the pleural surface, which was significantly lower than the $80 \%$ pneumothorax rate for lesions that did not contact the pleura $(P=0.04)$. Last, the development of postprocedure pneumothorax was associated with targeting smaller lesions $(P=0.03)$.

Statistical analysis demonstrated an incremental $8 \%$ increase in the risk of pneumothorax for every $10 \mathrm{~cm}^{3}$ decrease in lesion volume. A total of 11 fiducial markers (19\%) had migrated after placement. Fiducial migration was observed while targeting six lesions in six individual patients. The number of displaced fiducials ranged from one to five per patient (median 1). In four patients, displaced fiducials were found in the pleural space on postplacement CT images obtained within several minutes of insertion. In one patient, the fiducial remained within the lesion on the follow-up chest radiographs obtained up to $2 \mathrm{~h}$ after insertion. However, CT of the chest obtained
Fig. 2 Computed tomographic images demonstrating fiducial marker placement. The needle is inserted into the lesion (a, arrows), and the fiducial marker (arrowhead) is deposited within the middle of the lesion (b, arrows). In this case, there was a postprocedure pneumothorax (c, arrows), which was subsequently treated with a chest tube (d, arrows)

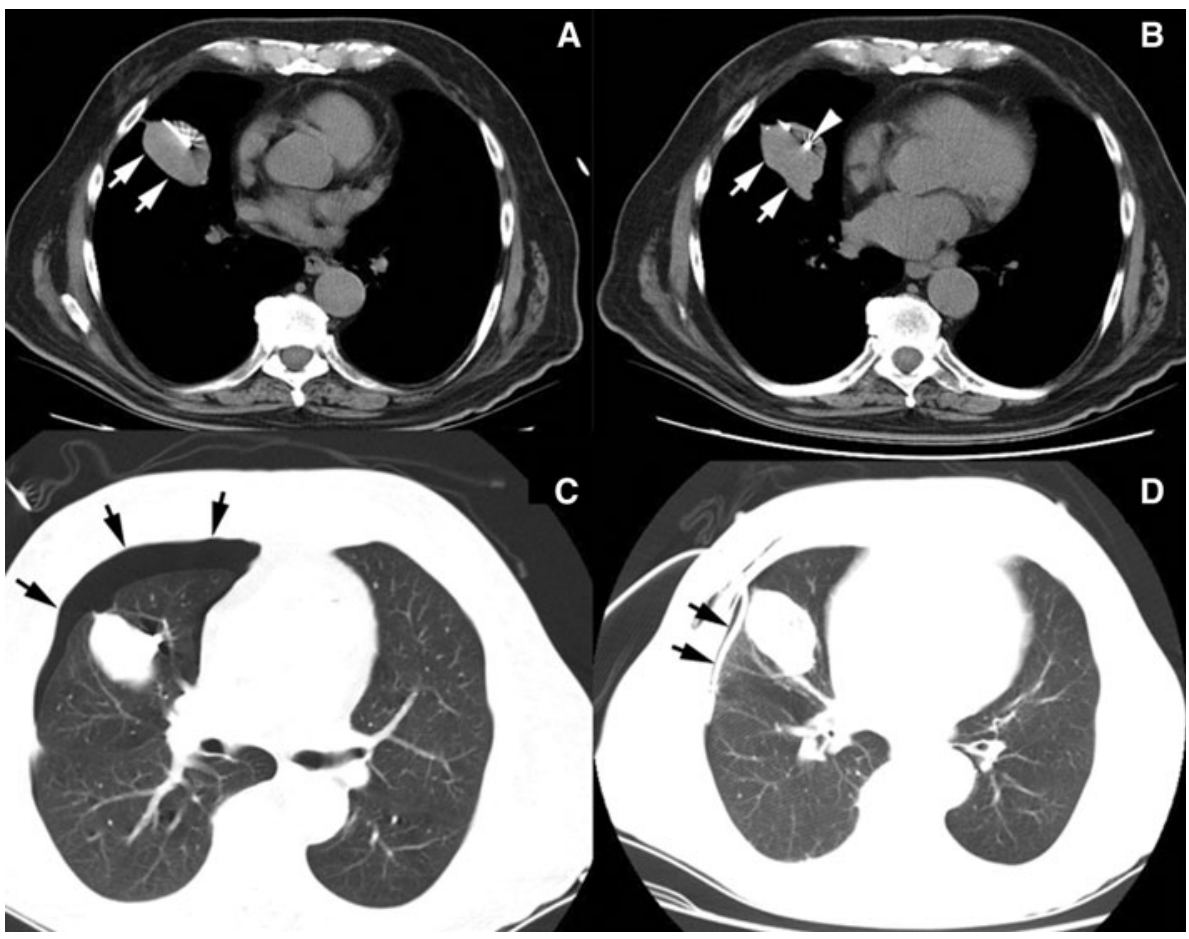


Table 1 Evaluation of potential risk factors for association with development of a pneumothorax (PTX)

\begin{tabular}{|c|c|c|c|c|c|}
\hline Factor & $\begin{array}{l}\text { Patients } \\
\text { without PTX }(n=9)\end{array}$ & $\begin{array}{l}\text { Patients with } \\
\text { PTX }(n=18)\end{array}$ & $\begin{array}{l}\text { PTX and chest tube } \\
(n=6)\end{array}$ & $P$ value $^{\mathrm{a}}$ & $P$ value ${ }^{\mathrm{b}}$ \\
\hline Lesion volume (mean $\pm \mathrm{SD}$ ) & $103 \pm 161 \mathrm{~cm}^{3}$ & $28 \pm 43 \mathrm{~cm}^{3}$ & $21 \pm 23 \mathrm{~cm}^{3}$ & 0.03 & 0.25 \\
\hline Needle trajectory length (mean $\pm \mathrm{SD}$ ) & $2.8 \pm 1.8 \mathrm{~cm}$ & $3.0 \pm 1.7 \mathrm{~cm}$ & $2.4 \pm 1.4 \mathrm{~cm}$ & 0.07 & 0.82 \\
\hline No. of markers, median (range) & $1(1-3)$ & $1(1-7)$ & $3(1-7)$ & 0.35 & 0.16 \\
\hline No. of needle adjustments, median (range) & $3(1-6)$ & $1(1-8)$ & $1(1-8)$ & 0.61 & 0.75 \\
\hline \multicolumn{6}{|l|}{ Lesion location, $n(\%)$} \\
\hline Upper lung zone & $2(20 \%)$ & $7(35 \%)$ & $3(50 \%)$ & 0.43 & 0.74 \\
\hline Lower lung zone & $8(80 \%)$ & $13(65 \%)$ & $3(50 \%)$ & & \\
\hline \multicolumn{6}{|l|}{ Lesion relationship to pleura, $n(\%)$} \\
\hline Contacts pleura & $6(60 \%)$ & $4(20 \%)$ & $1(17 \%)$ & 0.04 & 0.01 \\
\hline No pleural contact & $4(40 \%)$ & $16(80 \%)$ & $5(83 \%)$ & & \\
\hline Emphysema present, $n(\%)$ & $5(56 \%)$ & $5(28 \%)$ & $3(50 \%)$ & 0.19 & 0.83 \\
\hline Fissure crossed, $n(\%)$ & $0(0 \%)$ & $1(5 \%)$ & $1(17 \%)$ & 0.92 & 0.95 \\
\hline \multicolumn{6}{|l|}{ Needle used, $n(\%)$} \\
\hline 18 gauge & $3(33 \%)$ & $14(78 \%)$ & $5(71 \%)$ & 0.01 & 0.03 \\
\hline 19 gauge & $6(67 \%)$ & $4(22 \%)$ & $2(29 \%)$ & & \\
\hline
\end{tabular}

a Comparison between patients with and without PTX

${ }^{\mathrm{b}}$ Comparison between patients without PTX and patients with an enlarging or a symptomatic PTX that necessitated insertion of a chest tube

Fig. 3 Computed tomographic (CT) images demonstrating fiducial marker migration. Immediate postinsertion CT scan (a) showing the fiducial marker (arrowhead) within the lesion (arrows). On a scan 6 days later (b), the marker was not seen in the lesion (arrows). The marker was located in the costophrenic sulcus of the pleural space

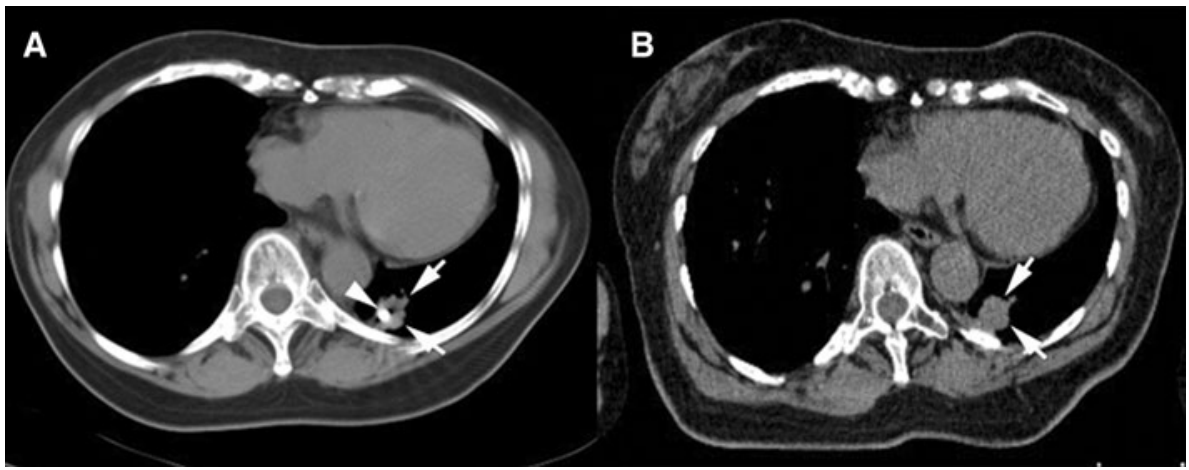

6 days after marker placement demonstrated that the fiducial had migrated into the pleural space (Fig. 3). This necessitated insertion of a second marker within the lesion. This fiducial remained within the nodule for at least 1 month after insertion, allowing the patient to receive a full course of radiosurgery. In the sixth patient, the marker was noted to have migrated into the contralateral airway immediately after placement. Subsequently, the patient experienced a bout of coughing, after which the marker was no longer seen on the CT images; it was likely expectorated.

Imaging follow-up with chest CT and/or chest radiographs was available for 1 to 46 months for all patients (mean 11.9 months). Fiducial marker migration was not detected beyond 6 days after placement. Statistical analysis (Table 2) demonstrated an association between the increased rate of fiducial migration and shorter distance from pleura to the marker deposition site $(P=0.04)$, as well as with fiducial placement outside of the target lesion $(P=0.03)$. No association was found between fiducial migration and lesion volume. In addition, fiducial migration was not associated with marker insertion through an iatrogenic pneumothorax.

\section{Discussion}

In this series, fiducial marker insertion in lung lesions resulted in a pneumothorax in $67 \%$ of patients. The rates of pneumothorax and subsequent chest tube insertion reported here are higher than those described in the literature (45 and 33\%, respectively; [6, 7]). We demonstrated increased rates of pneumothorax and chest tube insertion when 18-gauge guiding needles were used for fiducial marker insertion. Only 19-gauge needles were used for marker placement in other published series [6]. In a recent 
Table 2 Evaluation of potential risk factors for association with fiducial marker migration

\begin{tabular}{llll}
\hline Factor & $\begin{array}{l}\text { Migration } \\
(n=11)\end{array}$ & $\begin{array}{l}\text { No migration } \\
(n=48)\end{array}$ & $P$ value \\
\hline Lesion volume $\left(\mathrm{cm}^{3}\right)$ (mean $\left.\pm \mathrm{SD}\right)$ & $16 \pm 24$ & $54 \pm 102$ & 0.31 \\
Needle trajectory length $(\mathrm{cm})($ mean $\pm \mathrm{SD})$ & $1.4 \pm 1.4$ & $3.3 \pm 1.5$ & 0.04 \\
Needle tip location with respect to target lesion & & & \\
$\quad$ Inside lesion, $n(\%)$ & $1(9 \%)$ & $28(58 \%)$ & 0.03 \\
$\quad$ Outside lesion, $n(\%)$ & $10(91 \%)$ & $20(42 \%)$ & 0.92 \\
Pneumothorax before marker insertion, $\mathrm{n}(\%)$ & $7(64 \%)$ & $13(27 \%)$ & \\
\hline
\end{tabular}

large retrospective series of percutaneous lung biopsies, Geraghty et al. [8] also demonstrated a significantly higher risk of pneumothorax associated with the use of coaxial 18-gauge needles compared to 19-gauge needles. Use of a larger-bore needle may have led to a larger defect in the pleura. In addition, the 18-gauge needles may be stiffer and less prone to movement during respiration, leading to larger tears in the pleura. The analysis also showed that patients who developed a pneumothorax tended to have smaller lesions. Larger lesions may be more likely to extend to the pleura and allow needle passage into the lesion without traversing the aerated lung. We were not able to demonstrate that targeting smaller lesions required a larger number of needle adjustments within the lung parenchyma.

The pneumothorax rate after fiducial marker insertion reported here is also higher than the $8-64 \%$ rate of this complication after percutaneous lung biopsy [8, 9]. Furthermore, the $22 \%$ rate of chest tube insertion reported here is beyond the suggested threshold of $10 \%$ for lung biopsies according to the Quality Improvement Guidelines for Image-Guided Percutaneous Biopsy in Adults [10]. There are several potential reasons for these differences, including the use of 18-gauge needles (versus smaller needles for performing biopsies) and longer times that the needle remains in the lung parenchyma (as a result of the additional time required for fiducial marker insertion).

Fiducial migration after placement was encountered after insertion of $19 \%$ of the markers. Our analysis reveals that the rate of marker migration was higher when fiducials were deposited outside the target lesion and when the markers were placed in close proximity to the pleural surface. We found no association between fiducial migration and targeting smaller lesions or with marker insertion through an iatrogenic pneumothorax. Fiducials were most commonly dislodged at the time of or soon after insertion. Once an appropriate marker position was documented on the postplacement $\mathrm{CT}$, marker migration seemed uncommon.

Our study has several limitations. The study was retrospective, limiting the clinical information available for analysis. However, all preprocedure and follow-up imaging studies were available for review. In addition, the small sample size may have limited our ability to detect additional risk factors for development of a pneumothorax and for fiducial migration.

The rate of complications after fiducial marker insertion described in this study has serious implications. The $67 \%$ rate of pneumothorax, the $22 \%$ rate of chest tube insertion, and the $19 \%$ rate of seed migration are high. These complication rates may be reduced by altering the insertion technique or by changing the type of the fiducial marker used for lesion tracking. The dimensions of the currently used fiducial markers ( 1.2 by $3 \mathrm{~mm})$ mandate the use of at least a 19-gauge needle. A smaller marker size may make the insertion procedure safer.

Conflict of interest The authors declare that they have no conflict of interest.

Open Access This article is distributed under the terms of the Creative Commons Attribution Noncommercial License which permits any noncommercial use, distribution, and reproduction in any medium, provided the original author(s) and source are credited.

\section{References}

1. Uematsu M, Shioda A, Tahar K et al (1998) Focal, high dose, and fractionated modified stereotactic radiation therapy for lung carcinoma patients: a preliminary experience. Cancer 82:1062-1070

2. Murphy MJ (2004) Tracking moving organs in real time. Semin Radiat Oncol 14:91-100

3. Shirato H, Shimizu S, Kitamura K et al (2007) Organ motion in image-guided radiotherapy: lessons from real-time tumor-tracking radiotherapy. Int $\mathrm{J}$ Clin Oncol 12:8-16

4. Shirato J, Harada T, Harabayashi T et al (2003) Feasibility of insertion/implantation of $2.0 \mathrm{~mm}$ diameter gold internal fiducial markers for precise setup and real-time tumor tracking in radiotherapy. Int J Radiat Oncol Biol Phys 56:240-247

5. Collins J, Bloom A, Dai C et al (2007) CT-guided gold fiducial seed placement for respiratory gated external radiation therapy in thoracic and liver lesions. In: Presented at the annual meeting of the Cardiovascular and Interventional Radiological Society of Europe, Athens, Greece, September 8-12

6. Kothary N, Heit JJ, Louie JD et al (2009) Safety of percutaneous fiducial marker implantation for image-guided radiation therapy. J Vasc Interv Radiol 20:235-239

7. Yousefi S, Collins BT, Reichner CA et al (2007) Complications of thoracic computed tomography-guided fiducial placement for the purpose of stereotactic biopsy. Clin Lung Cancer 8:252-256 
8. Geraghty PR, Kee ST, McFarlane G et al (2003) CT-guided transthoracic needle aspiration biopsy of pulmonary nodules: needle size and pneumothorax rate. Radiology 229:475-481

9. Westcott JL (1998) Percutaneous transthoracic needle biopsy. Radiology 169:593-601
10. Cardella JF, Bakal CW, Bertino RE et al (2003) Quality improvement guidelines for image-guided percutaneous biopsy in adults. J Vasc Interv Radiol 14:S227-S230 